\title{
Compression of environmental representations following interactions with objects
}

\author{
Andrew Clement ${ }^{1}$ • Gabriel A. Radvansky ${ }^{1}$ - James R. Brockmole ${ }^{1}$
}

Published online: 21 August 2017

(C) The Psychonomic Society, Inc. 2017

\begin{abstract}
Previous work reveals that interacting with all objects in an environment can compress spatial memory for the entire group of objects. To assess the scope and magnitude of this effect, we tested whether interacting with a subset of objects compresses spatial memory for all objects in an environment. Participants inspected objects in one or two unmarked regions of space, then recalled the distances between pairs of objects from memory. One group of participants picked up objects in both regions, a second group picked up objects in one region and passively viewed objects in the other region, and a third group passively viewed objects in both regions. When participants manually interacted with objects, they recalled shorter object-pair distances throughout the environment. The magnitude of this effect was the same, regardless of whether participants interacted with all objects in the environment or just a subset of them. Together, these findings suggest that interacting with objects can compress environmental representations in memory, even when observers interact with a relatively small subset of objects.
\end{abstract}

Keywords Spatial memory · Environments · Interaction . Embodied cognition

In daily life, people often report distortions of visual perception. For example, athletes often describe the ball as unusually large, and people who look up at skyscrapers view them as impossibly tall. Although these cases are often dismissed as exaggeration, a growing body of evidence suggests that they

Andrew Clement

aclemen3@nd.edu

1 Department of Psychology, University of Notre Dame, Notre Dame, IN 46556, USA have a basis in perception. For example, athletes who are playing well actually perceive a larger ball or a wider goal (Witt \& Dorsch, 2010; Witt, Linkenauger, Bakdash, \& Proffitt, 2008; Witt \& Proffitt, 2005), and people who throw a heavy ball at a target perceive the target to be further away (Witt \& Proffitt, 2008; Witt, Proffitt, \& Epstein, 2004). People with a strong grip also perceive their hand to be larger (Linkenauger, Witt, Bakdash, Stefanucci, \& Proffitt, 2009), while people with broad shoulders perceive doorways to be narrower (Stefanucci \& Geuss, 2009). Similar distortions occur when people use hand-held tools. For example, when holding a reach-extending tool, people perceive distant objects to be closer (Bloesch, Davoli, Roth, Brockmole, \& Abrams, 2012; Davoli, Brockmole, \& Witt, 2012; Witt \& Proffitt, 2008; Witt, Proffitt, \& Epstein, 2005). Together, these findings support the action-specific account of perception, which states that perceptual features such as size and distance are scaled by one's physical abilities (Proffitt, 2006; Witt, 2011). Much like Gibson's (1979) theory of affordances, this account suggests that physical parameters play a critical role in perception, allowing people to view the world in terms of their own capacities for action.

One prominent feature of the action-specific account of perception is its focus on current physical demands. Because physical abilities change as a function of effort and intention, perception must be regularly updated to reflect one's current physical state (Proffitt, 2006; Witt, 2011). However, these action-specific distortions can also persist in memory, suggesting that perception is not only influenced by one's current abilities, but also by the physical demands of previous actions. For example, Vishton et al. (2007) found that reaching for the central circle within the Ebbinghaus illusion attenuated the perceived magnitude of the illusion. This attenuation persisted when participants completed a second block of trials without reaching, suggesting that manual interaction can produce distortions of perceptual memory. In another study, 
Davoli et al. (2012) found that participants who used a tool to interact with distant objects later recalled shorter distances to those objects. A number of studies have also found correlations between performance on physical tasks and the remembered sizes of taskrelevant objects (e.g., Wesp, Cichello, Gracia, \& Davis, 2004; Witt \& Proffitt, 2005; Witt etal., 2008). Together, these findings suggest that memory for size and distance can be influenced by physical parameters associated with action.

Most studies of action-specific distortions have focused on interactions with individual objects. In such cases, only the targets of interaction appear to be distorted in memory. For example, Cañal-Bruland and van der Kamp (2009) had children throw a ball at a target, then asked them to estimate the size of the target. In this case, children who successfully hit the target recalled the target to be larger. However, when children successfully caught a ball that was launched from the target's location, they recalled the ball to be larger, but no longer overestimated the size of the target. Thus, the size of the target was only distorted when it was relevant to participants' current action goals. However, people do not always interact with one object at a time, but instead interact with multiple objects in their surroundings. In such cases, it is unclear how interacting with more than one object might affect memory for the entire group of objects. To begin to address this issue, Thomas, Davoli, and Brockmole (2013) had participants examine spatial layouts containing multiple objects. Participants walked to each object, then inspected each one by either picking it up or passively viewing it. After manually or visually inspecting all objects, participants entered a separate room and recalled the objects' locations from memory. When participants manually inspected the objects, they recalled shorter object-pair distances than when they visually inspected the objects, revealing compressed memory for the spatial layout of objects. Thus, when all objects were relevant to participants' action goals, actionspecific distortions were observed for the entire group of objects.

Given these findings, several questions can be raised about the scope of action-specific distortions. First, what happens when observers only interact with a subset of objects in an environment? In Thomas etal.'s (2013) study, participants interacted with all objects in the environment. However, people do not always interact with all objects in their surroundings, but instead interact with specific subsets of objects. In such cases, it is unclear how interacting with a subset of objects might affect memory for other objects in the environment. On one hand, this situation could resemble cases in which people interact with individual objects, with interaction only distorting memory for the targets of interaction. This would suggest that objects' locations are stored independently in memory, with actions only distorting memory for action-relevant objects (Cañal-Bruland \& van der Kamp, 2009). Alternatively, this situation could resemble cases in which people interact with all objects in an environment, with interaction distorting memory for all objects in the environment. This would suggest that actions distort memory for entire environments, not just for objects that are currently action-relevant. Suggestive evidence for this position comes from Thomas et al.'s (2013) study, where participants who manually inspected objects not only recalled shorter distances between the objects, but also between four cones that marked the boundaries of the spatial layout. Because participants never interacted with these boundary markers, this suggests that action-specific distortions may extend to objects that are not directly interacted with. However, because participants never directly inspected the boundary markers, this finding may simply reflect a response bias. It is possible that when all objects in an environment are inspected, other objects in an environment may serve as landmarks, anchoring memory for the targets of interaction (Holyoak \& Mah, 1982; McNamara \& Diwadkar, 1997; Sadalla, Burroughs, \& Staplin, 1980). In this case, interacting with a subset of objects would not be expected to distort memory for any objects in the environment. Such an outcome would be consistent with several recent studies that have failed to replicate action-specific distortions (e.g., Durgin et al., 2009).

Assuming interacting with a subset of objects distorts spatial memory for all objects in an environment, an important secondary question concerns the magnitude of these distortions. Specifically, does the magnitude of these distortions remain the same when observers interact with all objects or just a subset of them? Although action-specific distortions may occur for all objects in an environment, the magnitude of these distortions may differ depending on whether objects are directly interacted with. For example, memory for the targets of interaction may be strongly distorted, while memory for other objects in the environment may be distorted less strongly. In this case, the magnitude of action-specific distortions would depend on an object's action relevance, with action-relevant objects being distorted more strongly in memory (Cañal-Bruland \& van der Kamp, 2009). Alternatively, memory for all objects in the environment may be strongly distorted, regardless of whether the objects are directly interacted with. In this case, the magnitude of action-specific distortions would not be dependent on action relevance, but would instead be driven by distortion of the entire environment. Another possibility is that memory for all objects may be distorted less strongly than when all objects in the environment are interacted with. In this case, the presence of other objects would anchor memory for the targets of interaction, attenuating any action-specific distortions (Holyoak \& Mah, 1982; McNamara \& Diwadkar, 1997; Sadalla et al., 1980).

In summary, the present study had two main goals. First, we sought to identify whether interacting with a subset of objects compresses spatial memory for all objects in an environment. Second, assuming spatial compression is observed for all objects, we sought to identify whether the magnitude of compression is consistent throughout an environment. By addressing these issues, the present study provided important information about the scope and magnitude of action-specific distortions. 


\section{Experiment 1}

In Experiment 1, we sought to conceptually replicate Thomas et al.'s (2013) finding that interacting with all objects in an environment compresses memory for the spatial layout of objects. In their study, participants drew scale maps or reconstructed the object layouts from memory. While both of these methods were sensitive to action-specific distortions, each has important limitations. Maps are easily generated, but require mental transformations of scale. As a result, they only provide information about the relative distances between objects. Reconstructed object layouts avoid these mental transformations, but are costly in terms of research time and space. To avoid these limitations, we asked participants to provide numerical estimates of distance (e.g., "How far apart were objects $x$ and $y$ ?"). We chose this method because it is a common way of assessing spatial memory, both in the laboratory and in everyday settings (Montello, 1991). Importantly, numerical distance estimates are easily generated and provide precise measures of distance. They are also sensitive to actionspecific distortions (e.g., Bloesch et al., 2012; Davoli et al., 2012; Witt \& Proffitt, 2008; Witt et al., 2004, 2005), making them ideal for assessing compression of spatial memory.

\section{Method}

Participants A group of 85 University of Notre Dame undergraduates participated for $\$ 10$ or course credit. Seven participants were removed from analysis because they did not follow instructions or entered responses incorrectly when estimating distance (e.g., omitted decimal points on a large proportion of trials).

Apparatus and stimuli The experiment was conducted in a $4.6 \times 4.9-\mathrm{m}$ room. Five objects (scissors, bowl, cup, hanger, foam finger) were placed within the room at locations and orientations held constant across participants (see Fig. 1).

Procedure and design Participants were instructed to walk to each object, study it for $10 \mathrm{~s}$, and memorize its details for a future memory test. They were not explicitly instructed to memorize the objects' locations. An experimenter prompted participants when to move to a new object, as well as which objects to move to. Each participant examined the objects in a unique, randomly determined order. Participants in the manual condition ( $n=38$ ) picked up each object, studied it, and replaced it in its original location before proceeding to the next object. Participants in the visual condition $(n=40)$ passively viewed the objects from any angle but did not touch them. After inspecting all objects, participants entered a separate room and completed a computer-based memory test. On each trial, a pair of object names appeared in the center of the screen, and participants recalled the distance between the objects (the default measure was feet). All possible object pairs

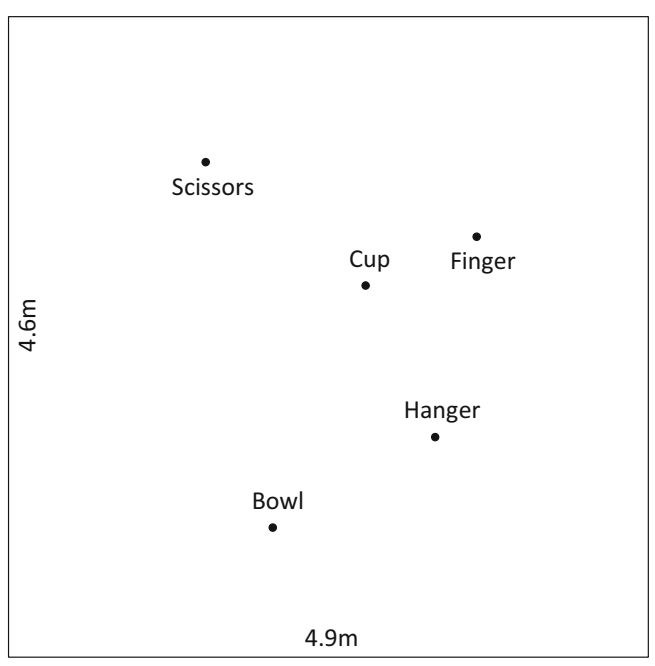

Fig. 1 The object layout used in Experiment 1

were presented in a random order. Each pair appeared in both of two possible orders (e.g., scissors-hanger and hanger-scissors), resulting in a total of 20 trials.

\section{Results}

Prior to analysis, all object-pair distances were converted to meters. To test whether spatial compression occurred, we compared average object-pair distances for the manual and visual conditions using an independent-samples $t$ test. ${ }^{1}$ The analysis revealed that participants in the manual condition $(M=1.08 \mathrm{~m}, S D=0.26 \mathrm{~m})$ recalled shorter object-pair distances than those in the visual condition $(M=1.25 \mathrm{~m}, S D=$ $0.43 \mathrm{~m}), t(76)=2.07, p=.042, \eta_{p}{ }^{2}=.053$. This replicates Thomas et al.'s (2013) findings, revealing compression of spatial memory when participants interacted with all objects in an environment.

\section{Discussion}

The results of Experiment 1 revealed compression of spatial memory when participants interacted with all objects in an environment. ${ }^{2}$ Specifically, participants who manually

\footnotetext{
${ }^{1}$ To assess the internal consistency of participants' distance estimates, we also correlated the estimated object-pair distances for both orders of a pair. Overall, participants' distance estimates for one order (e.g., scissors-hanger) correlated positively with estimates for the other order (e.g., hanger-scissors), $r=.69, p<$ .001 . These correlations did not differ between the manual and visual conditions, $p=.752$.

${ }^{2}$ Here, we are referring to relative compression of spatial memory, rather than participants' tendency to underestimate distances from memory (e.g., Hubbard, 1994). Indeed, when we compared participants' distance estimates to the actual object-pair distances, we found that participants in both the manual (mean difference $=-0.50 \mathrm{~m}$ ), $p<.001$, and visual conditions (mean difference $=-0.34 \mathrm{~m}), p<.001$, significantly underestimated distance. However, participants in the manual condition also recalled shorter distances than those in the visual condition. Thus, following interaction with objects, memory for the objects' locations was compressed relative to when participants did not interact with objects.
} 
interacted with objects recalled shorter object-pair distances than those who passively viewed objects. Having demonstrated this effect, our second experiment tested whether spatial compression could extend to other objects in an environment.

\section{Experiment 2}

In Experiment 2, we sought to assess whether interacting with a subset of objects compresses memory for the spatial layout of all objects in an environment. Participants inspected objects in two unmarked regions of space, then recalled the distances between all object pairs. One group of participants picked up objects in both regions, a second group picked up objects in one region and passively viewed objects in the other region, and a third group passively viewed objects in both regions. If compression occurs for objects in both regions, this would suggest that physical interaction distorts memory throughout an environment (Thomas et al., 2013). However, if compression is limited to objects in one region, this would suggest that interaction only distorts memory for action-relevant objects (Cañal-Bruland \& van der Kamp, 2009). Moreover, if compression is not observed in either region, this would suggest that the presence of other objects anchors memory for the targets of interaction (Holyoak \& Mah, 1982; McNamara \& Diwadkar, 1997; Sadalla et al., 1980). By comparing participants who manually inspected objects in one or both regions, we also sought to assess whether the magnitude of compression remains the same when observers interact with all objects or just a subset of them. If the magnitude of compression is the same in both cases, this would suggest that physical interaction strongly distorts memory throughout an environment. However, if compression is attenuated for objects in one region, this would suggest that the magnitude of action-specific distortions depends on the action relevance of objects. Moreover, if compression is attenuated for objects in both regions, this would suggest that the presence of other objects reduces the magnitude of action-specific distortions.

\section{Method}

Participants A new group of 95 University of Notre Dame undergraduates participated for $\$ 10$ or course credit. Five participants were removed from analysis because they did not follow instructions or entered responses incorrectly when estimating distance.

Apparatus and stimuli The experiment was conducted in the same room as in Experiment 1. The same five objects were placed within an unmarked $2.7 \times 3.1-\mathrm{m}$ region in the center of the room (inner region). The locations of these objects were the same as in Experiment 1. However, five additional objects (tape, binder, flying disc, pliers, stapler) were placed at new locations outside this region (outer region). There was no visible division between the two regions. All objects were placed at locations and orientations held constant across participants (see Fig. 2).

Procedure and design The task was similar to Experiment 1. Participants studied each object for $10 \mathrm{~s}$ and memorized its details. An experimenter prompted each participant to examine the objects in a unique, randomly determined order, with participants inspecting all objects in one region before proceeding to the next one. The order of inspection was counterbalanced across participants, with half of participants studying the inner objects first and half studying the outer objects first. Participants in the all-manual condition $(n=$ 29) picked up each object, studied it, and replaced it in its original location before proceeding to the next object. Participants in the half-manual condition $(n=29)$ picked up each object in the inner region, but only passively viewed the objects in the outer region. Participants in the visual condition $(n=32)$ passively viewed the objects from any angle but did not touch them. After inspecting all objects, participants entered a separate room and completed the memory test from Experiment 1. All possible object pairs were presented in a random order, and participants recalled the distances between object pairs. Each pair appeared in both of two possible orders, resulting in a total of 90 trials. Of these trials, 20 contained objects from the inner region (inner region pairs), 20 contained objects from the outer region (outer region pairs), and 50 contained objects from both regions (across region pairs).

\section{Results}

Prior to analysis, all object-pair distances were converted to meters. To test for order effects, we first analyzed average object-pair distances using a 2 (order: inner region first, outer region first) $\times 3$ (inspection: all-manual, half-manual, visual) $\times 3$ (pair type: inner region, outer region, across region) mixed-model analysis of variance (ANOVA). ${ }^{3}$ Order and inspection were entered as between-subjects variables, and pair type was entered as a within-subjects variable. No significant effects of order were observed, $p$ s for all order effects $\geq .276$. As a result, we collapsed across levels of order and ran a 3 (inspection: all-manual, half-manual, visual) $\times 3$ (pair type: inner region, outer region, across region) mixed-model ANOVA. Unsurprisingly, there was a significant main effect of pair type, $F(2,174)=273.14, p<.001, \eta_{p}{ }^{2}=.758$. Pairwise comparisons revealed that participants recalled shorter

\footnotetext{
3 To assess the internal consistency of participants' distance estimates, we again correlated the estimated object-pair distances for both orders of a pair As in Experiment 1, participants' distance estimates for one order correlated positively with estimates for the other order, $r=.65, p<.001$. These correlations did not differ between the three inspection conditions, $p=.195$.
} 


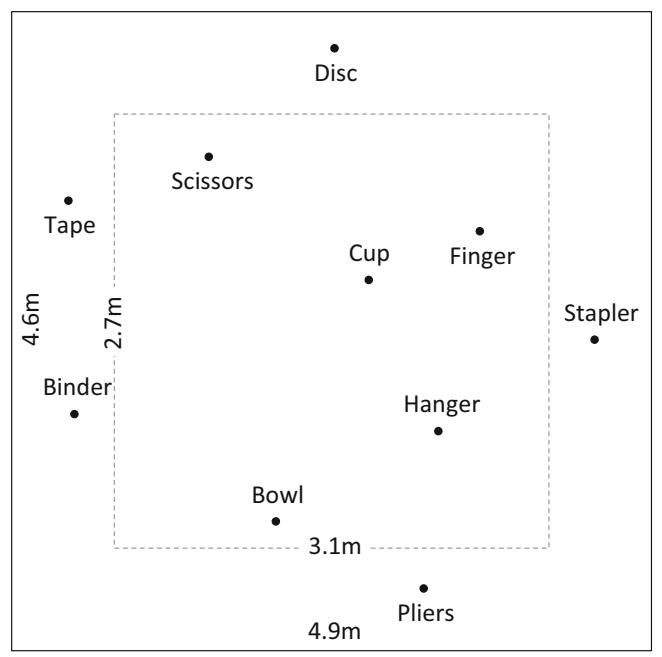

Fig. 2 The object layout used in Experiment 2. The dotted line represents the regional boundary, which was not visible to participants

distances for inner region pairs $(M=1.12 \mathrm{~m}, S D=0.34 \mathrm{~m})$ than for outer region pairs $(M=1.69 \mathrm{~m}, S D=0.45 \mathrm{~m}), p<$ .001 , and across region pairs $(M=1.36 \mathrm{~m}, S D=0.35 \mathrm{~m}), p<$ .001. Participants likewise recalled shorter distances for across region pairs than for outer region pairs, $p<.001$. There was also a significant main effect of inspection, $F(2,87)=3.84, p$ $=.025, \eta_{p}{ }^{2}=.081$. Pairwise comparisons revealed that participants in both the all-manual $(M=1.31 \mathrm{~m}, S D=0.39 \mathrm{~m}), p=$ .016 , and half-manual conditions $(M=1.32 \mathrm{~m}, S D=0.34 \mathrm{~m})$, $p=.023$, recalled shorter distances than those in the visual condition $(M=1.53 \mathrm{~m}, S D=0.32 \mathrm{~m})$. However, there was no significant difference between the all-manual and half-manual conditions, $p=.894$. Thus, the magnitude of spatial compression did not differ between the two conditions. Importantly, there was no significant interaction between inspection and pair type, $F(1,59)=1.74, p=.181$. Thus, participants in both the all-manual and half-manual conditions recalled shorter object-pair distances throughout the environment (see Fig. 3).

\section{Discussion}

The results of Experiment 2 revealed that spatial compression occurred for all objects in an environment, even when observers interacted with only a subset of objects. ${ }^{4}$ Although participants in the half-manual condition only interacted with objects in the inner region, they recalled shorter object-pair

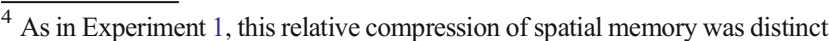
from participants' tendency to underestimate distances from memory. When we compared participants' distance estimates to the actual object-pair distances, we found that participants in the all-manual (mean difference $=$ $-0.85 \mathrm{~m}$ ), $p<.001$, half-manual (mean differences $=-0.85 \mathrm{~m}$ ), $p<.001$, and visual conditions (mean difference $=-0.64 \mathrm{~m}$ ), $p<.001$, all significantly underestimated distance. However, participants in both the all-manual and half-manual conditions recalled shorter distances than those in the visual condition, revealing further compression of spatial memory when participants interacted with objects.
}

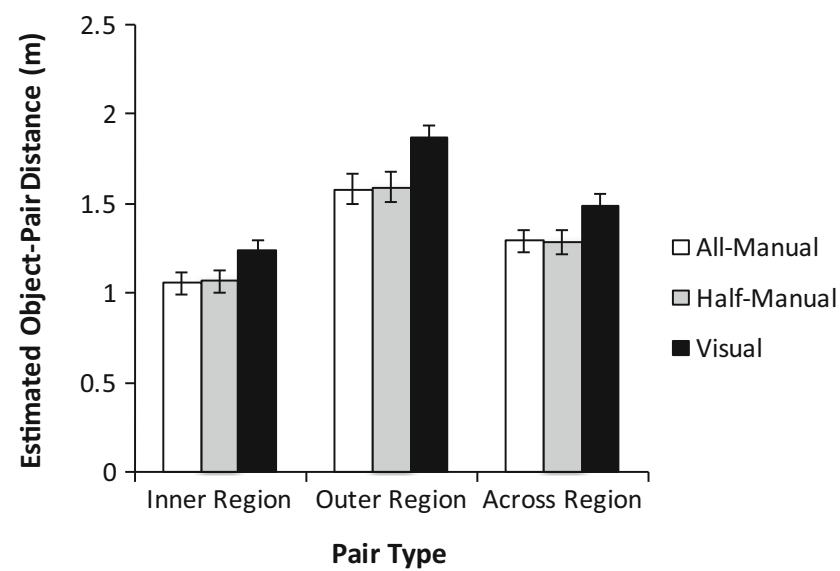

Fig. 3 Average object-pair distances for Experiment 2. Error bars reflect \pm 1 standard error

distances throughout the environment. The magnitude of this effect was the same in the all-manual condition, where participants interacted with objects in both regions. Thus, physical interaction strongly distorted memory for all objects in the environment, not just for the targets of interaction. Together, these findings suggest that interacting with a relatively small subset of objects can compress spatial memory for entire environments.

\section{General discussion}

Previous work reveals that interacting with all objects in an environment can compress spatial memory for the entire group of objects (Thomas et al., 2013). While this finding suggests that actions can affect spatial memory for multiple objects, the full extent of this effect is unknown. To address this issue, the present study assessed the scope and magnitude of action-specific distortions. In everyday circumstances, people rarely interact with all objects in an environment, but instead interact with specific subsets of objects. In such cases, do action-specific distortions extend throughout an environment, or are they limited to objects that are directly interacted with? If physical interaction distorts memory for all objects in the environment, this would suggest that actionspecific distortions occur at the level of entire environments (Thomas et al., 2013). However, if physical interaction only distorts memory for the targets of interaction, this would suggest that action-specific distortions occur at the level of individual objects (Cañal-Bruland \& van der Kamp, 2009). Moreover, if actionspecific distortions do not occur, this would suggest that the presence of other objects anchors memory for the targets of interaction (Holyoak \& Mah, 1982; McNamara \& Diwadkar, 1997; Sadalla et al., 1980). Assuming action-specific distortions are observed for all objects, does the magnitude of these distortions remains the same when observers interact with all objects or just a subset of them? If the magnitude of these distortions is the same in both cases, this would suggest that actins strongly distort memory 
throughout an environment. However, if these distortions are attenuated for objects that are not directly interacted with, this would suggest that the effects of interaction depend on the action relevance of objects. Moreover, if these distortions are attenuated for all objects in the environment, this would suggest that the presence of other objects reduces the effects of interaction.

Overall, our findings provide evidence that physically interacting with objects compresses memory for the spatial layout of objects. In Experiment 1, participants who manually interacted with a group of objects recalled shorter object-pair distances than those who passively viewed the objects. This replicates Thomas et al.'s (2013) findings, revealing compression of spatial memory when participants interacted with all objects in an environment. More importantly, Experiment 2 revealed that interacting with a subset of objects compressed spatial memory for all objects in an environment. In this case, participants who manually interacted with a subset of objects recalled shorter object-pair distances throughout the environment than those who passively viewed them. The magnitude of this effect was the same, regardless of whether participants interacted with all objects in the environment or just a subset of them. Thus, physical interaction strongly distorted memory for all objects, not just those that were directly interacted with. This result is consistent with Thomas et al.'s (2013) findings, which suggested that spatial compression might extend to objects that are not interacted with. Thus, unlike cases in which people interact with individual objects (Cañal-Bruland \& van der Kamp, 2009), interacting with a relatively small group of objects can affect spatial memory for entire environments.

Although we interpret these findings as evidence that physical interaction influences spatial memory, there are several alternative explanations for these results. For example, because participants in the manual condition bent over to pick up the objects, one could argue that manually inspecting objects required more physical effort than visually inspecting them. Indeed, physical effort has been shown to influence the perceived distances of objects (Proffitt, Stefanucci, Banton, \& Epstein, 2003; Witt \& Proffitt, 2008; Witt et al., 2004). Because participants in the manual condition held the objects in their hands, one could also argue that manually inspecting objects involved viewing them from a closer distance. While both physical effort and viewing distance could have influenced participants' spatial memory, we consider both of these explanations unlikely. Although picking up objects may have required additional effort, participants in both the manual and visual conditions bent over while inspecting objects. In fact, because participants were instructed to view the objects from any angle, participants in the visual condition often knelt down to inspect objects from multiple angles. Thus, both physical effort and viewing distance were roughly equated in the manual and visual conditions. It is also worth noting that Thomas et al. (2013) found no added effects of physical effort or viewing distance when participants visually inspected objects. Thus, while both physical effort and viewing distance may have played a role in the present findings, it is more likely that these findings were due to physical interaction with objects.

More broadly, the present findings add to a list of known factors that influence spatial memory. As a large body of research indicates, memory for spatial locations is influenced by the structure of the surrounding environment (e.g., Stevens \& Coupe, 1978). For example, observers underestimate the distance between objects located in the same environment, but overestimate the distances between objects located in different environments (Hirtle \& Jonides, 1985; McNamara, 1986; McNamara, Hardy, $\&$ Hirtle, 1989). Spatial memory is also influenced by the presence of landmarks and other reference points, with observers underestimating the distance from landmarks to nearby objects (Holyoak \& Mah, 1982; McNamara \& Diwadkar, 1997; Sadalla et al., 1980; see also Nelson \& Chaiklin, 1980). These findings are consistent with many theoretical models of spatial memory, which suggest that memory for spatial locations is biased by surrounding objects and regions (e.g., Huttenlocher, Hedges, \& Duncan, 1991). The present findings suggest that interacting with objects not only influences memory for the locations of individual objects, but also for the layout of the surrounding environment. This suggests that observers' interactions with the environment play a greater role in spatial memory than many theoretical accounts assume. Nonetheless, the present study only examined how interacting with a central group of objects influences memory for surrounding regions. Future research should examine these effects under other circumstances, such as when objects are located in adjacent regions. Indeed, research suggests that these regions may be represented differently in memory (Brockmole \& Wang, 2002; Wang \& Brockmole, 2003).

In summary, the present study provided important information about the scope and magnitude of action-specific distortions. In two experiments, we demonstrated that interacting with a subset of objects compresses spatial memory throughout an environment. The magnitude of this compression was the same, regardless of whether observers interacted with all objects in the environment or just a subset of them. Together, these findings suggest that physical interaction can compress environmental representations in memory, even when observers only interact with a relatively small subset of objects.

\section{References}

Bloesch, E. K., Davoli, C. C., Roth, N., Brockmole, J. R., \& Abrams, R. A. (2012). Watch this! Observed tool use affects perceived distance. Psychonomic Bulletin \& Review, 19(2), 177-183.

Brockmole, J. R., \& Wang, R. F. (2002). Switching between environmental representations in memory. Cognition, 83(3), 295-316.

Cañal-Bruland, R., \& van der Kamp, J. (2009). Action goals influence action-specific perception. Psychonomic Bulletin \& Review, 16(6), $1100-1105$. 
Davoli, C. C., Brockmole, J. R., \& Witt, J. K. (2012). Compressing perceived distance with remote tool-use: Real, imagined, and remembered. Journal of Experimental Psychology: Human Perception and Performance, 38(1), 80-89.

Durgin, F. H., Baird, J. A., Greenburg, M., Russell, R., Shaughnessy, K., \& Waymouth, S. (2009). Who is being deceived? The experimental demands of wearing a backpack. Psychonomic Bulletin \& Review, 16(5), 964-969.

Gibson, J. J. (1979). The ecological approach to visual perception. Boston, MA: Houghton Mifflin.

Hirtle, S. C., \& Jonides, J. (1985). Evidence of hierarchies in cognitive maps. Memory \& Cognition, 13(3), 208-217.

Holyoak, K. J., \& Mah, W. A. (1982). Cognitive reference points in judgments of symbolic magnitude. Cognitive Psychology, 14(3), 328-352.

Hubbard, T. L. (1994). Memory psychophysics. Psychological Research, $56(4), 237-250$.

Huttenlocher, J., Hedges, L. V., \& Duncan, S. (1991). Categories and particulars: Prototype effects in estimating spatial location. Psychological Review, 98(3), 352-376.

Linkenauger, S. A., Witt, J. K., Bakdash, J. Z., Stefanucci, J. K., \& Proffitt, D. R. (2009). Asymmetrical body perception: A possible role for neural body representations. Psychological Science, 20(11), $1373-1380$.

McNamara, T. P. (1986). Mental representations of spatial relations. Cognitive Psychology, 18(1), 87-121.

McNamara, T. P., \& Diwadkar, V. A. (1997). Symmetry and asymmetry of human spatial memory. Cognitive Psychology, 34(2), 160-190.

McNamara, T. P., Hardy, J. K., \& Hirtle, S. C. (1989). Subjective hierarchies in spatial memory. Journal of Experimental Psychology: Learning, Memory, and Cognition, 15(2), 211-227.

Montello, D. R. (1991). The measurement of cognitive distance: Methods and construct validity. Journal of Environmental Psychology, 11(2), $101-122$.

Nelson, T. O., \& Chaiklin, S. (1980). Immediate memory for spatial location. Journal of Experimental Psychology: Human Learning and Memory, 6(5), 529-545.

Proffitt, D. R. (2006). Embodied perception and the economy of action. Perspectives on Psychological Science, 1(2), 110-122.

Proffitt, D. R., Stefanucci, J., Banton, T., \& Epstein, W. (2003). The role of effort in perceiving distance. Psychological Science, 14(2), 106112 .
Sadalla, E. K., Burroughs, J. W., \& Staplin, L. J. (1980). Reference points in spatial cognition. Journal of Experimental Psychology: Human Learning and Memory, 6(5), 516-528.

Stefanucci, J. K., \& Geuss, M. N. (2009). Big people, little world: The body influences size perception. Perception, 38(12), 1782-1795.

Stevens, A., \& Coupe, P. (1978). Distortions in judged spatial relations. Cognitive Psychology, 10(4), 422-437.

Thomas, L. E., Davoli, C. C., \& Brockmole, J. R. (2013). Interacting with objects compresses environmental representations in spatial memory. Psychonomic Bulletin \& Review, 20(1), 101-107.

Vishton, P. M., Stephens, N. J., Nelson, L. A., Morra, S. E., Brunick, K. L., \& Stevens, J. A. (2007). Planning to reach for an object changes how the reacher perceives it. Psychological Science, 18(8), 713719.

Wang, R. F., \& Brockmole, J. R. (2003). Simultaneous spatial updating in nested environments. Psychonomic Bulletin \& Review, 10(4), 981986.

Wesp, R., Cichello, P., Gracia, E. B., \& Davis, K. (2004). Observing and engaging in purposeful actions with objects influences estimates of their size. Perception \& Psychophysics, 66(8), 1261-1267.

Witt, J. K. (2011). Action's effect on perception. Current Directions in Psychological Science, 20(3), 201-206.

Witt, J. K., \& Dorsch, T. E. (2010). Kicking to bigger uprights: Field goal kicking performance influences perceived size. Perception, 38(9), $1328-1340$.

Witt, J. K., Linkenauger, S. A., Bakdash, J. Z., \& Proffitt, D. R. (2008). Putting to a bigger hole: Golf performance relates to perceived size. Psychonomic Bulletin \& Review, 15(3), 581-585.

Witt, J. K., \& Proffitt, D. R. (2005). See the ball, hit the ball: Apparent ball size is correlated with batting average. Psychological Science, 16(12), 937-938.

Witt, J. K., \& Proffitt, D. R. (2008). Action-specific influences on distance perception: A role for motor simulation. Journal of Experimental Psychology: Human Perception and Performance, 34(6), 1479-1492.

Witt, J. K., Proffitt, D. R., \& Epstein, W. (2004). Perceiving distance: A role of effort and intent. Perception, 33(5), 577-590.

Witt, J. K., Proffitt, D. R., \& Epstein, W. (2005). Tool use affects perceived distance, but only when you intend to use it. Journal of Experimental Psychology: Human Perception and Performance, $31(5), 880-888$. 